\title{
Clinical Value of Noninducibility by High-Dose Isoproterenol Versus Rapid Atrial Pacing After Catheter Ablation of Paroxysmal Atrial Fibrillation
}

\author{
THOMAS CRAWFORD, M.D., AMAN CHUGH, M.D., ERIC GOOD, D.O., \\ KENTARO YOSHIDA, M.D., KRIT JONGNARANGSIN, M.D., MATTHEW EBINGER, D.O., \\ FRANK PELOSI JR., M.D., FRANK BOGUN, M.D., FRED MORADY, M.D., \\ and HAKAN ORAL, M.D. \\ From the Division of Cardiovascular Medicine, University of Michigan, Ann Arbor, Michigan, USA
}

Noninducibility by High-Dose Isoproterenol. Objective: To determine the relative clinical value of noninducibility of atrial fibrillation (AF) by isoproterenol (ISO) and by rapid atrial pacing (RAP) in patients with paroxysmal AF (PAF).

Background: AF can be induced by RAP or ISO in $>85 \%$ of patients with PAF.

Methods: ISO was administered in escalating doses of $5,10,15$, and $20 \mu \mathrm{g} / \mathrm{min}$ in 112 patients (age $=56 \pm$ 13 years) with PAF before radiofrequency catheter ablation. AF was inducible in 97 of 112 patients $(87 \%)$ at a mean dose of $15 \pm 5 \mu \mathrm{g} / \mathrm{min}$. RAP induced AF in the remaining 14 of 15 patients. Antral pulmonary vein (PV) isolation (APVI) was followed by ablation of complex fractionated atrial electrograms (CFAEs) as necessary to terminate $\mathrm{AF}$ and render AF noninducible in response to ISO.

Results: AF terminated during APVI in 72 of 111 patients (65\%) and after APVI plus ablation of CFAEs in 11 of 111 patients $(10 \%)$. In the remaining 28 patients $(25 \%)$, sinus rhythm was restored by transthoracic cardioversion. RAP was performed in the last 61 consecutive patients who were rendered noninducible by ISO. RAP initiated AF in 20 of 61 patients $(33 \%)$ and atrial flutter in 6 patients $(10 \%)$. No additional ablation was performed if AF was induced with RAP; however, atrial flutter was targeted. At $12 \pm 5$ months, 63/75 patients $(84 \%)$ who were noninducible by ISO and 2 of $8(25 \%)$ who still were reinducible by ISO were free from recurrent AF after a single ablation procedure without antiarrhythmic drugs $(\mathbf{P}=$ $0.001)$. AF recurred in 20 of 36 patients $(56 \%)$ who required cardioversion for persistent $\mathrm{AF}$ after ablation $(P<0.001)$. Among the 61 patients who also underwent RAP, 12 of $20(60 \%)$ who were, and 31 of $41(76 \%)$ who were not inducible by $R A P$ were free from recurrent $A F(P=0.21)$. The accuracy of noninducibility as a predictor of clinical outcome was $83 \%$ with ISO and $64 \%$ by $\operatorname{RAP}(P=0.03)$.

Conclusions: The response to isoproterenol after catheter ablation of PAF more accurately predicts clinical outcome than the response to RAP. (J Cardiovasc Electrophysiol, Vol. 21, pp. 13-20, January 2010)

atrial fibrillation, catheter ablation, isoproterenol, atrial pacing, pulmonary vein isolation

In patients with paroxysmal atrial fibrillation $(\mathrm{AF})$, the noninducibility of AF by rapid atrial pacing after catheter ablation identifies patients who are less likely to have recurrent $\mathrm{AF}$ during follow-up than those who remain in $\mathrm{AF}$ or still have inducible $\mathrm{AF}^{1-3}$ However, even when AF is still inducible by rapid pacing at the end of an ablation procedure, $>50 \%$ of patients remain free of AF during follow-up, indicating that the $\mathrm{AF}$ that is induced by rapid pacing sometimes is a nonspecific response.

A recent study demonstrated that isoproterenol has high sensitivity and specificity for the induction of AF in pa-

Drs. Oral and Morady are founders of Ablation Frontiers, Inc., and have consulted to Ablation Frontiers, Inc. No other conflicts of interest were declared.

Address for correspondence: Hakan Oral, M.D., Cardiovascular Center, SPC 5853, 1500 East Medical Center Drive, Ann Arbor, MI 48109-5853, USA. Fax: +1-734-936-7026; E-mail: oralh@umich.edu

Manuscript received 15 March 2009; Revised manuscript received 30 June 2009; Accepted for publication 7 July 2009.

doi: $10.1111 / \mathrm{j} .1540-8167.2009 .01571 . x$ tients with paroxysmal $\mathrm{AF}^{4}$ Therefore, it is possible that isoproterenol is a more accurate predictor than rapid pacing of clinical outcomes after ablation. However, no prior studies have compared the clinical value of isoproterenol versus rapid atrial pacing as predictors of outcomes after catheter ablation of paroxysmal AF.

The purpose of this study was to prospectively determine whether the noninducibility of AF in response to isoproterenol is predictive of freedom from recurrent $\mathrm{AF}$ after catheter ablation and whether the predictive value of isoproterenol is higher or lower than that of rapid atrial pacing.

\section{Methods}

\section{Study Subjects}

The subjects of this prospective study were 112 consecutive patients who presented to the electrophysiology laboratory in sinus rhythm for catheter ablation of paroxysmal AF (PAF). There were 75 men and 37 women. The mean age of the patients was $56 \pm 13$ years. AF was first diagnosed $7 \pm 6$ years before presentation. The mean left ventricular ejection 
fraction and left atrial diameter were $0.60 \pm 0.07$ and $40 \pm$ $6 \mathrm{~mm}$, respectively. Structural heart disease was present in 27 patients: hypertensive heart disease in 17, coronary artery disease in 6, and valvular heart disease in 4.

Patients with uncontrolled hypertension, obstructive coronary artery disease with inducible ischemia, hypertrophic cardiomyopathy, and aortic or mitral stenosis were excluded from this study. Among the 112 patients 18 had undergone a prior ablation procedure for AF.

\section{Electrophysiological Study}

All patients provided written informed consent. The electrophysiology procedures were performed in the fasting state. All antiarrhythmic drugs, except for amiodarone in 5 patients, were discontinued at least $4-5$ half lives before the procedure. Amiodarone was discontinued 8 weeks before the ablation procedure. Vascular access was obtained through a femoral vein. A quadripolar catheter was placed in the coronary sinus for recording electrograms and for atrial pacing. After transseptal catheterization, systemic anticoagulation was achieved with intravenous heparin to maintain an activated clotting time of 300-350 seconds. A decapolar ring catheter (Lasso, Biosense Webster, Diamond Bar, CA, USA) was used to map the pulmonary veins (PVs). Bipolar electrograms were displayed and recorded at filter settings of 30 to $500 \mathrm{~Hz}$ (EPMed Systems). The esophagus was visualized by barium swallow as described previously. ${ }^{5}$ After the barium swallow, conscious sedation was achieved with fentanyl and midazolam.

\section{Study Protocol}

The study protocol was approved by the Institutional Review Board. Before ablation, isoproterenol was infused at escalating doses of $5,10,15$, and $20 \mu \mathrm{g} / \mathrm{min}$ for 2 minutes at each infusion rate, as described previously. ${ }^{4}$ Isoproterenol was discontinued when AF was induced, if there was a decrease in systolic blood pressure to $<85 \mathrm{mmHg}$, if the patient complained of severe chest tightness, or if ischemic changes were noted on an electrocardiogram. Episodes of AF that occurred during the first 15 minutes of isoproterenol washout were considered to have been induced by isoproterenol. In patients who did not have inducible AF in response to isoproterenol, AF was induced by rapid atrial pacing from the coronary sinus.

Radiofrequency catheter ablation was performed as described below to terminate and render AF noninducible in response to isoproterenol. In patients who had termination of AF during ablation, isoproterenol was readministered using the same infusion protocol. If AF was inducible in response to isoproterenol, additional mapping was performed to target residual foci. Frequent atrial ectopy in the form of bursts of atrial tachycardia or atrial bigeminy/trigeminy in response to isoproterenol was also targeted. If AF was not terminated by ablation and electrical cardioversion had to be performed, the patient was not rechallenged with isoproterenol.

Rapid atrial pacing was also performed in the last 61 consecutive patients who were rendered noninducible in response to isoproterenol. The atrium was paced from the coronary sinus at a cycle length of $220 \mathrm{~ms}$ down to the cycle length at which there was loss of 1:1 atrial capture, 5 times for 10 seconds each. An episode of AF $\geq 60$ seconds was considered sustained and indicated inducibility of AF. In patients with sustained AF in response to rapid atrial pacing, no fur- ther ablation was performed and sinus rhythm was restored by pharmacologic or transthoracic cardioversion. Sustained atrial flutter or atrial tachycardia induced by either rapid atrial pacing or high dose isoproterenol was targeted for ablation. In order to assess the specificity of the response to rapid atrial pacing, no additional ablation was performed if AF was induced by rapid atrial pacing. Sinus rhythm was restored in these patients by transthoracic cardioversion.

\section{Catheter Ablation}

Antral pulmonary vein isolation (APVI) was performed to isolate all PVs during AF, using an open-irrigation, 3.5-mmtip deflectable catheter (Thermocool, Biosense Webster). PV isolation was confirmed by entrance block and absence of PV potentials. If AF still persisted after APVI, complex fractionated atrial electrograms (CFAEs) in the left atrium and coronary sinus were targeted. CFAEs were defined as fractionated electrograms with $\geq 2$ deflections, continuous electrical activity without an isoelectric interval, or electrograms with a cycle length $\leq 120 \mathrm{~ms}^{6}{ }^{6}$ The extent of CFAE ablation and the endpoint of AF termination were at the discretion of the operator. A 3-D electroanatomical mapping system (CARTO, Biosense Webster) was used to reduce fluoroscopy exposure and to facilitate catheter navigation. Radiofrequency energy was delivered at power settings of $25-35 \mathrm{~W}$, with maximum temperature of $45^{\circ} \mathrm{C}$ at flow rates of $17-30 \mathrm{~mL} / \mathrm{min}$.

\section{Post-Ablation Management and Clinical Follow-Up}

All patients underwent electrocardiographic monitoring during an overnight hospital stay. Patients were discharged home taking warfarin and low-molecular weight heparin, which was discontinued once the INR was $\geq 2.0$. Warfarin was discontinued at 3 months and aspirin was substituted unless there was another indication for anticoagulant therapy or unless the patient had a history of stroke/transient ischemic episode.

All patients were seen in an outpatient clinic 3 months after the procedure and every 3-6 months thereafter. Patients were provided with an auto-trigger event monitor for 21-30 days at 6 months after the procedure. Any episode of $A F \geq 30$ seconds was considered to be a recurrence. Patients were also asked to call whenever they experienced symptoms suggestive of an arrhythmia. In addition, patients were interviewed by telephone every 4-6 months to assess their symptomatic status.

The primary clinical endpoint of this study was freedom from symptomatic and asymptomatic AF in the absence of antiarrhythmic drug therapy after a single ablation procedure. Recurrences of other atrial arrhythmias also were reported.

\section{Statistical Analysis}

Continuous variables are expressed as mean \pm standard deviation and were compared using Student $t$-test. Categorical variables were compared by chi-square analysis or with the Fisher exact test, as appropriate. A P $<0.05$ indicated statistical significance.

\section{Results}

\section{Inducibility of AF Prior to Ablation}

Isoproterenol induced sustained AF in 97 of 112 patients (87\%, Fig. 1). In 8 additional patients (7\%), frequent 
A

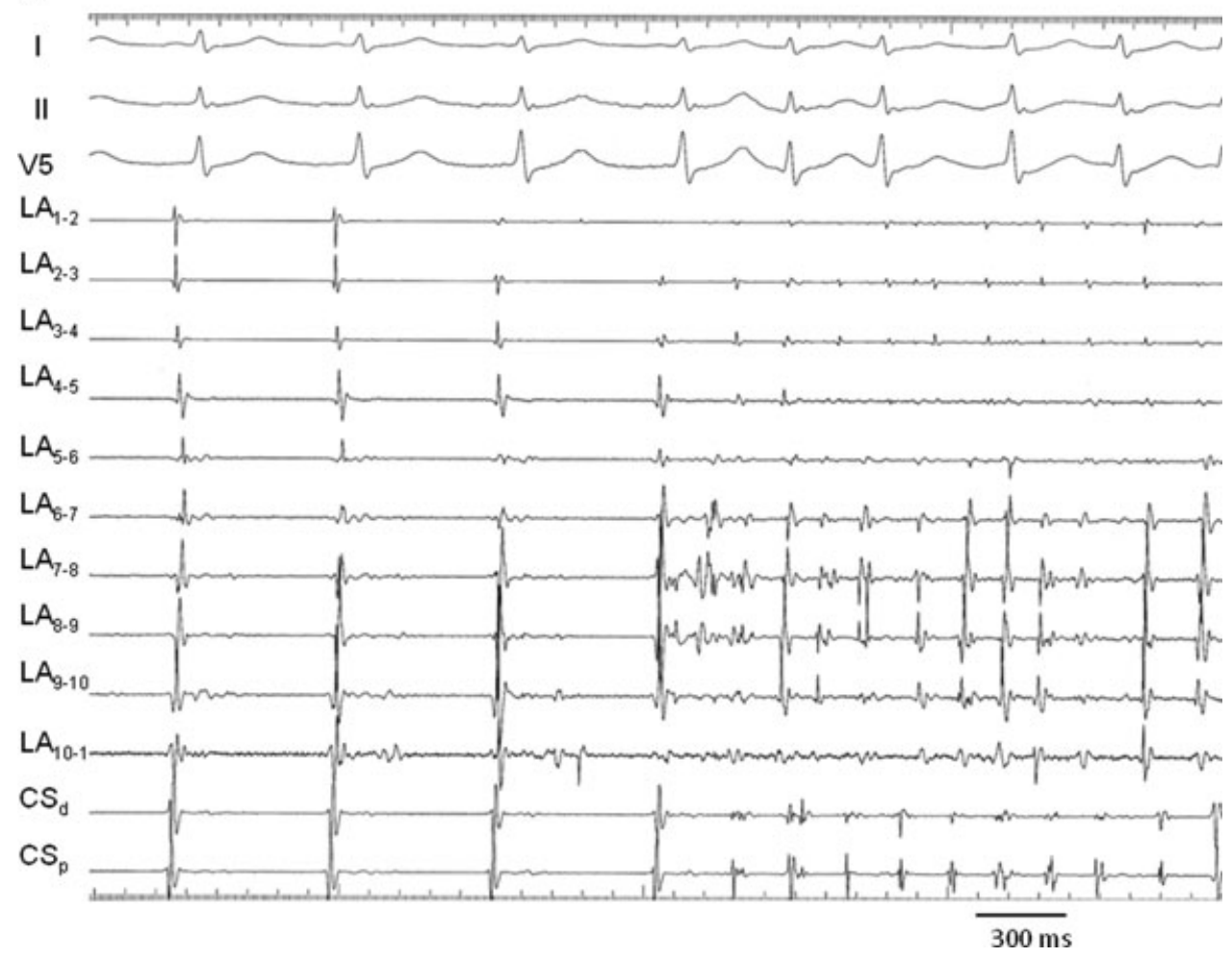

B

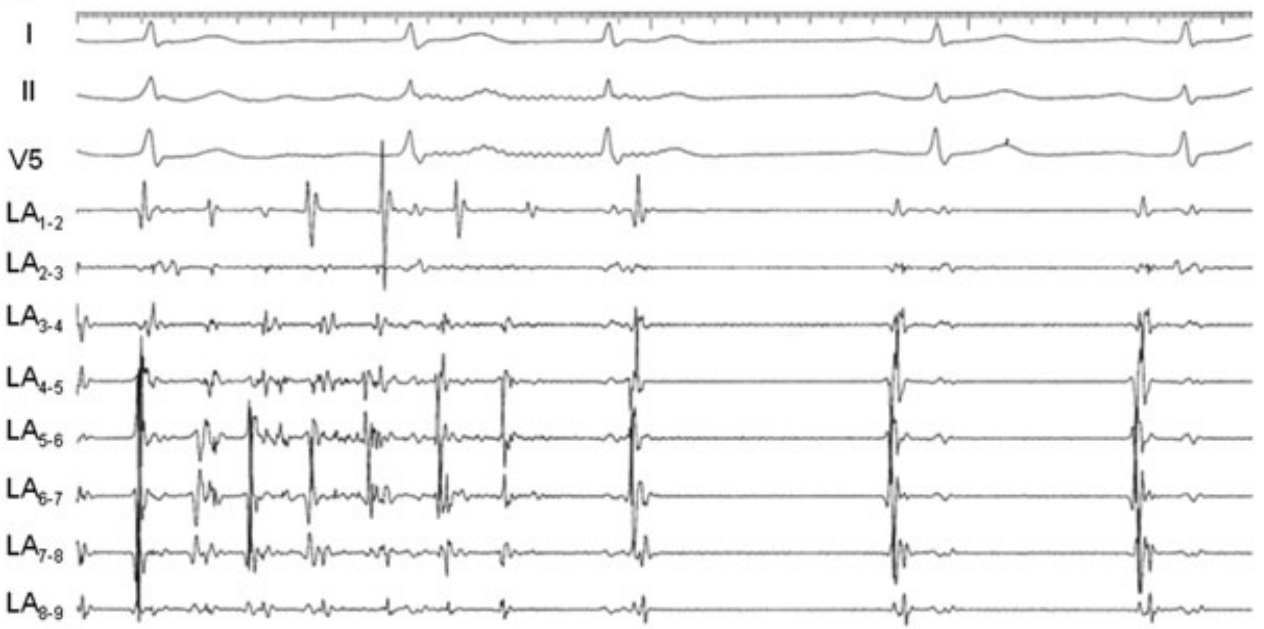

$\mathrm{LA}_{9.10}$

$\mathrm{LA}_{10.1}$

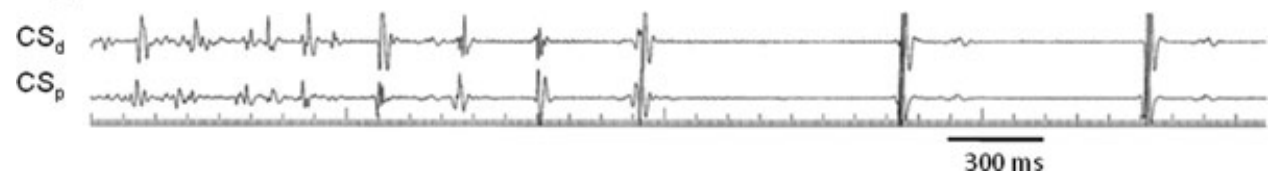

Figure 1. Initiation of AF during infusion of isoproterenol at $15 \mu \mathrm{g} / \mathrm{min}$ (Panel A). Shown are leads I, II, and $V_{5}$, and electrograms recorded from the bipoles of a decapolar ring catheter positioned at the ostium of the left superior $P V\left(L A_{1-2}\right.$ to $\left.L A_{10-1}\right)$ and a quadripolar catheter in the coronary sinus $\left(C S_{d}\right.$ and $C S_{p}$ : distal and proximal bipoles of the coronary sinus catheter). During APVI around this vein, AF terminated (Figure 1B) and subsequently the vein was completely isolated. Isoproterenol infusion at $20 \mu \mathrm{g} / \mathrm{min}$ did not induce AF after APVI (not shown).

premature atrial depolarizations (4), short bursts of AF (3), or sustained atrial tachycardia (1) were induced by isoproterenol. In the remaining 7 patients (6\%), no atrial arrhythmia was inducible with isoproterenol. Among these 7 patients the target dose of $20 \mu \mathrm{g} / \mathrm{min}$ was reached in 4 , and in the remain- ing 3 patients isoproterenol was discontinued before reaching the maximum dose because of chest pain in 2 and ventricular tachycardia in 1 . The mean dose of isoproterenol that induced $\mathrm{AF}$ was $15 \pm 5 \mu \mathrm{g} / \mathrm{min}$. AF was induced by rapid atrial pacing in 14 of 15 patients in whom sustained AF was 


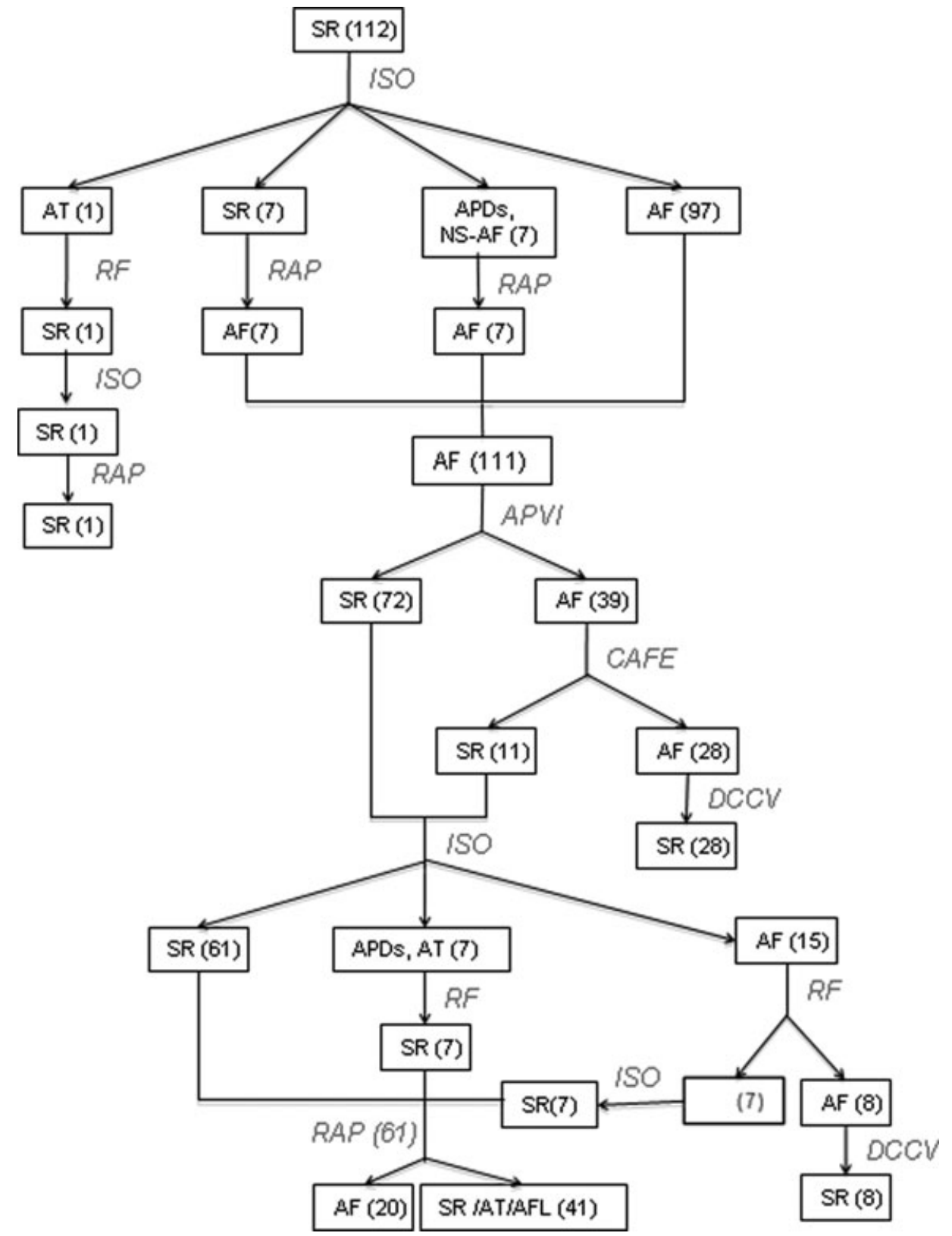

Figure 2. Study flow diagram. $A F=$ atrial fibrillation; $A F L=$ atrial flutter; $A P D s=$ frequent atrial premature depolarizations; $A P V I=$ antral pulmonary vein isolation; $A T=$ atrial tachycardia; $C F A E=$ complex atrial fractionated electrogram; $D C C V=$ direct current cardioversion; $I S O=$ isoproterenol infusion; $N S-A F=$ nonsustained $A F ; R A P=$ rapid atrial pacing; $R F A=$ radiofrequency $a b$ lation; $S R=$ sinus rhythm. not induced by isoproterenol. In 1 patient, only sustained atrial tachycardia originating in a PV was inducible despite infusion of isoproterenol and rapid atrial pacing. Because AF could not be induced, this patient was excluded from subsequent analyses.

\section{Catheter Ablation}

APVI resulted in isolation of all PVs. The mean duration of radiofrequency energy application was $45 \pm 13 \mathrm{~min}$. AF terminated during APVI in 72 of 111 patients with inducible AF $(65 \%)$, including 64 of 97 patients $(66 \%)$ in whom AF had been induced by isoproterenol and 8 of 14 patients $(57 \%)$ in whom AF had been induced by rapid atrial pacing $(\mathrm{P}=$ 0.73).

Among the 39 patients who remained in AF after APVI, ablation of CFAEs resulted in termination of AF in $11(28 \%)$, including 9 of 34 patients $(26 \%)$ in whom AF had been induced by isoproterenol and 2 of 5 patients $(40 \%)$ in whom AF had been induced by rapid atrial pacing $(\mathrm{P}=0.6)$. The mean duration of radiofrequency energy application for ablation of CFAEs was $7 \pm 6 \mathrm{~min}$. Overall, APVI with or without ablation of CFAEs terminated AF in 83 of 111 patients $(75 \%)$. In the remaining 28 patients $(25 \%)$ sinus rhythm was restored by cardioversion (Fig. 2).

\section{Reinduction of AF by Isoproterenol After Ablation}

AF was reinduced by isoproterenol in 15 of 83 patients $(18 \%)$ in whom AF terminated during ablation, including 14 of 76 patients $(18 \%)$ in whom AF had been terminated by APVI and 1 of 7 patients (14\%) in whom AF had been terminated by additional ablation of CFAEs $(\mathrm{P}=1.0$, Fig. 3). Additional ablation at the PV ostia (5) or left atrial roof (2) terminated AF and rendered it noninducible by isoproterenol in 7 of these 15 patients $(47 \%)$. In addition, isoproterenol rechallenge resulted in frequent premature depolarizations or atrial tachycardia in 7 patients 


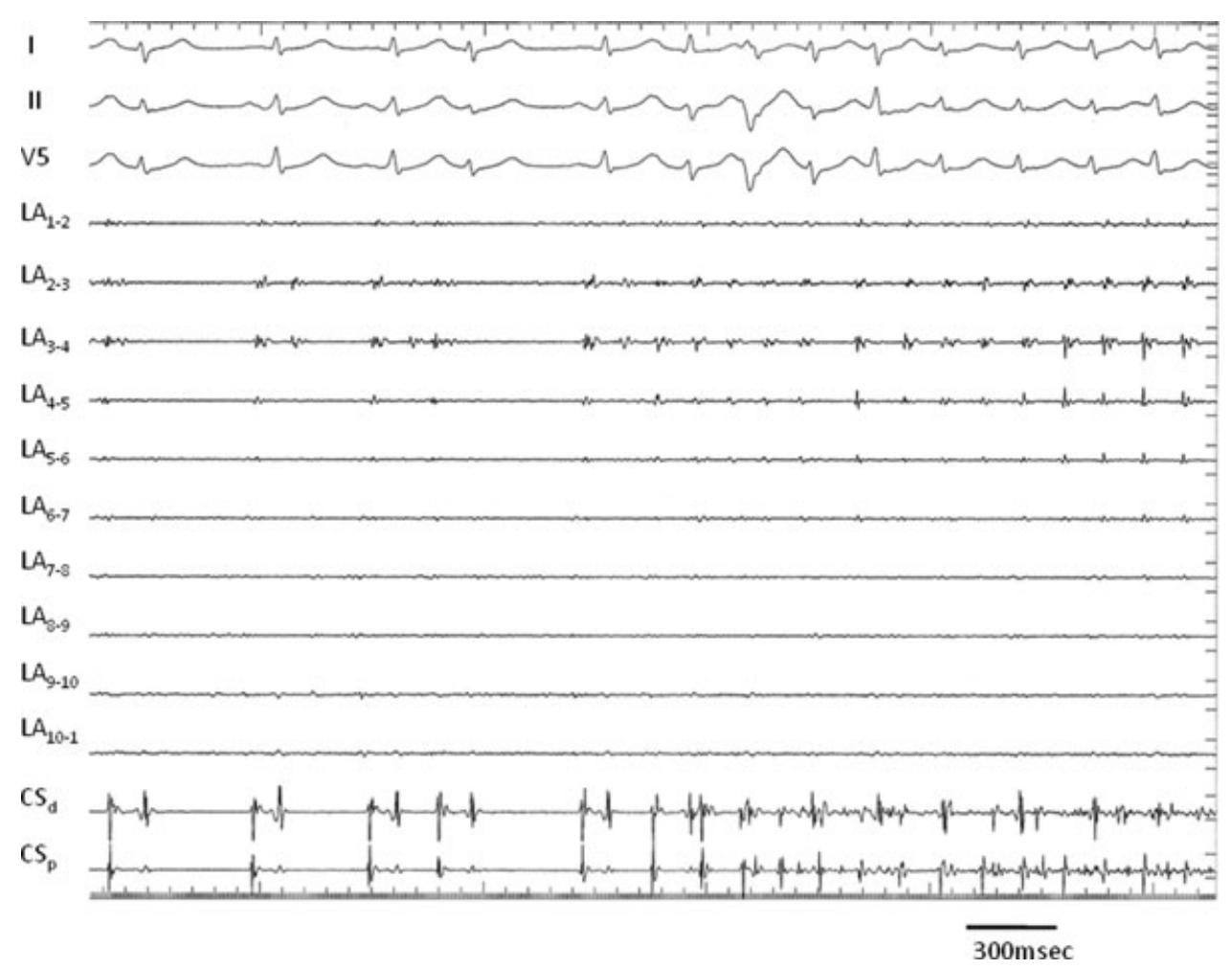

Figure 3. Reinducibility of $A F$ by isoproterenol rechallenge. AF was induced by isoproterenol infusion at $10 \mu g / m i n$ before the ablation. Termination of AF in this patient occurred during ablation at the antrum of the right-sided PVs. AF was reinducible by isoproterenol infusion at $15 \mu \mathrm{g} /$ min. Further mapping demonstrated a residual focus in the right inferior PV. Additional radiofrequency ablation resulted in termination of AF. Isoproterenol at $20 \mu \mathrm{g} /$ min did not reinduce AF (not shown). This patient remained free from recurrent atrial arrhythmias during follow-up. Abbreviations as in Figure 1.

(Fig. 2). All of these arrhythmias were rendered noninducible after additional ablation. Sinus rhythm was restored by transthoracic cardioversion in 8 of 15 patients in whom AF that was induced during isoproterenol rechallenge could not be terminated by ablation. In these 8 patients the additional ablation was performed along the posterior mitral annulus and inferior wall (5), along the rim between the left-sided PVs and left atrial appendage (4), PV ostia (2), base of left atrial appendage (2), and anterior left atrium (2).

After ablation, AF terminated and was rendered noninducible in response to isoproterenol in 75 of $111(68 \%)$, including 69 of 97 patients (71\%) who had been inducible by isoproterenol and 6 of $14(43 \%)$ who had required rapid atrial pacing to induce $\mathrm{AF}(\mathrm{P}=0.04)$.

\section{Inducibility of AF by Rapid Atrial Pacing After Catheter Ablation}

Rapid atrial pacing induced sustained AF in 20 of 61 (33\%) and sustained atrial flutter in another 6 of 61 patients (10\%, Fig. 4). The flutter was successfully ablated in all of the 6 patients. In accord with the study design, no additional ablation was performed in the patients with inducible $\mathrm{AF}$ and sinus rhythm was restored by cardioversion.

\section{Recurrent AF}

At a mean follow-up of $12 \pm 5$ months, 79 of 111 patients (71\%) were free from recurrent AF after a single ablation procedure in the absence of antiarrhythmic drug therapy. Among the 111 patients, 69 of 97 (71\%) who had inducible $\mathrm{AF}$ by isoproterenol before ablation and 10 of $14(71 \%)$ who required rapid atrial pacing for induction of $\mathrm{AF}$ before ablation were free from recurrent $\mathrm{AF}(\mathrm{P}=0.98)$. Among the 111 patients, 60 of 84 patients $(71 \%)$ who underwent only APVI and 19 of 27 patients who also had ablation of CFAEs (70\%) were free from recurrent $\mathrm{AF}(\mathrm{P}=0.92)$.

\section{Clinical Outcome and Noninducibility by Isoproterenol Versus Rapid Atrial Pacing}

Among the 111 patients, 63 of 75 (84\%) who were rendered noninducible in response to isoproterenol were free from recurrent AF during follow-up, compared with 2 of 8 patients (25\%) who had inducible AF during isoproterenol rechallenge and required cardioversion despite additional ablation to terminate and render $\mathrm{AF}$ noninducible $(\mathrm{P}=0.001$, Fig. 5). Among the 28 patients who remained in $\mathrm{AF}$ after ablation and therefore required electrical cardioversion for restoration of sinus rhythm (and who were not rechallenged with isoproterenol), 14 of 28 (50\%) remained free from recurrent $\mathrm{AF}(\mathrm{P}<0.001$ compared with patients who were rendered noninducible). Overall, 63 of 75 patients (84\%) who were rendered noninducible in response to isoproterenol and 16 of 36 patients (44\%) who still were reinducible by isoproterenol and/or required cardioversion after ablation due to persistent $\mathrm{AF}$, remained free from recurrent $\mathrm{AF}$ after a single ablation procedure without antiarrhythmic drug therapy $(\mathrm{P}<0.0001)$.

Among the 61 patients who were noninducible by isoproterenol and were challenged with rapid atrial pacing, 31 of $41(76 \%)$ who were not inducible by rapid atrial pacing were free from recurrent AF. Freedom from AF was not significantly different $(12 / 20,60 \%)$ among the patients who did 

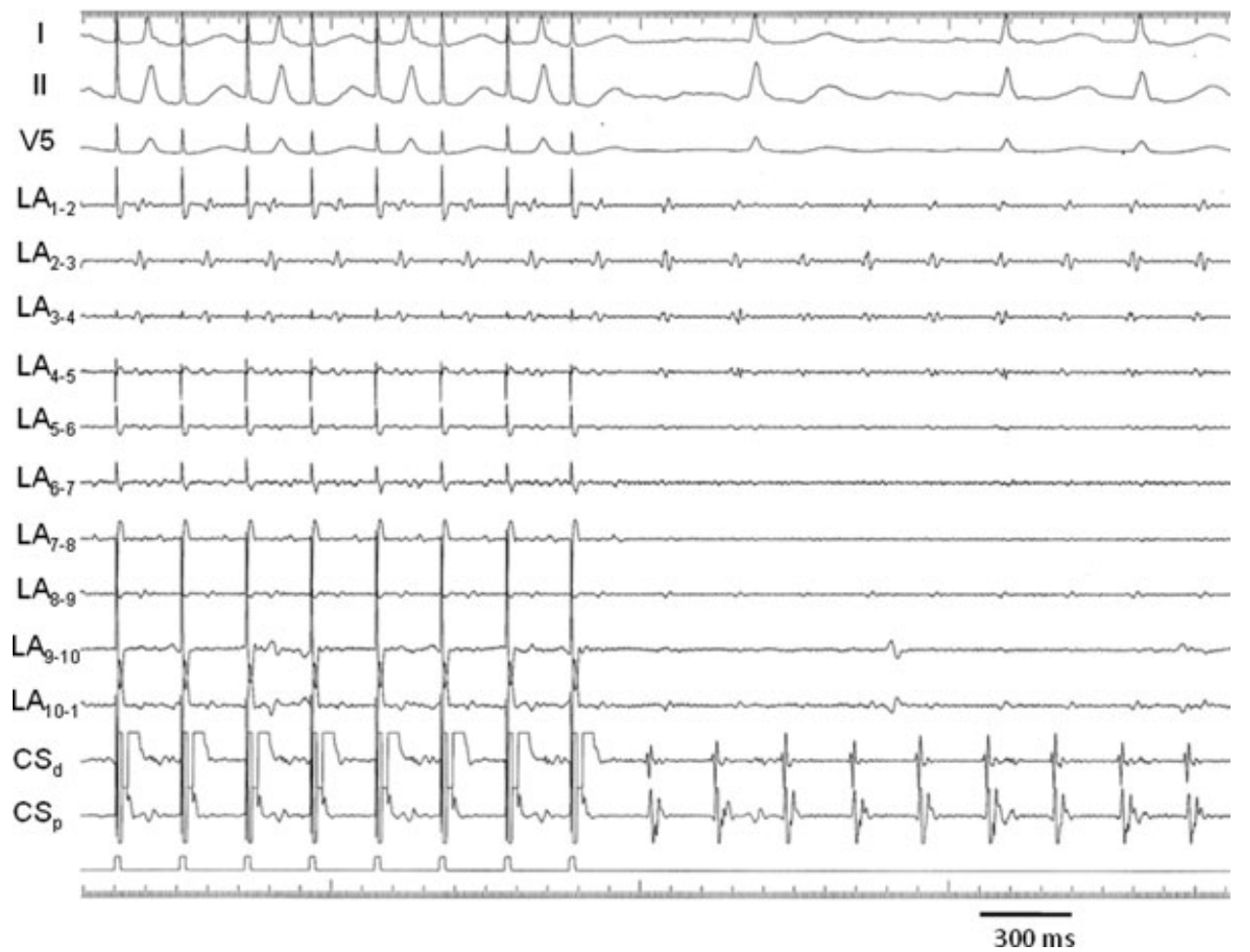

Figure 4. Induction of atrial flutter by rapid atrial pacing. Isoproterenol infusion at $20 \mu \mathrm{g} / \mathrm{min}$ failed to induce AF or atrial flutter. However, rapid atrial pacing induced sustained atrial flutter utilizing the mitral isthmus. Abbreviations as in Figure 1.

have inducible $\mathrm{AF}$ in response to rapid atrial pacing $(\mathrm{P}=$ 0.21 , Fig. 5).

\section{Atrial Tachycardia}

Atrial tachycardia occurred during follow-up in 7 of 102 (7\%) and 3 of 9 patients (33\%) who were and were not rendered noninducible in response to isoproterenol after the

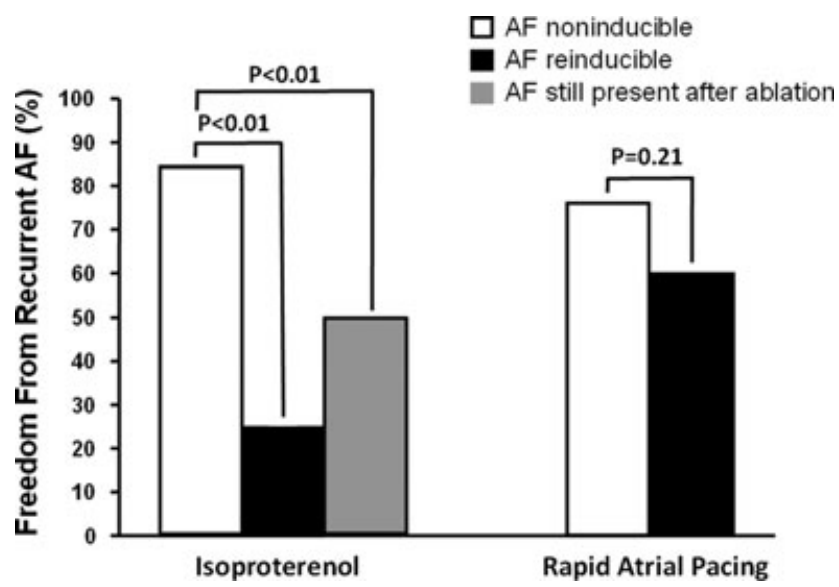

Figure 5. Proportion of patients who were free from recurrent AF after catheter ablation. Patients who were rendered noninducible (open bars) in response to isoproterenol had a more favorable outcome than patients whose $A F$ was reinducible during isoproterenol rechallenge despite additional ablation (black bar). Patients who remained in AF after ablation and required transthoracic cardioversion to attain sinus rhythm (gray bar) also had a less favorable outcome than patients who were rendered noninducible. The clinical outcome of patients who did not (open bar) and who did (black bar) have inducible AF by rapid atrial pacing also is shown. ablation procedure $(\mathrm{P}=0.03)$. The prevalence of atrial tachycardia was 6 of $84(7 \%)$ in patients who underwent only APVI and 4 of $28(14 \%)$ in patients who also had ablation of CFAEs $(\mathrm{P}=0.3)$.

\section{Predictive Accuracy}

The sensitivity and specificity of post-ablation isoproterenol-induced AF for recurrent AF during followup were $33 \%$ and $97 \%$, respectively. The positive and negative predictive values for AF reinducible by isoproterenol after the ablation to predict freedom from recurrent AF were $75 \%$ and $84 \%$, respectively. The overall diagnostic accuracy was $83 \%$ (Table 1). The sensitivity and specificity of rapid atrial pacing for recurrent AF during follow-up were $44 \%$ and $72 \%$, respectively. The positive and negative predictive values were $40 \%$ and $76 \%$, respectively. The overall diagnostic accuracy of rapid atrial pacing-induced AF was $64 \%$ $(\mathrm{P}=0.03$ compared with isoproterenol, Table 1$)$.

TABLE 1

Accuracy of Isoproterenol and Rapid Atrial Pacing in Predicting Recurrences of AF After Catheter Ablation

\begin{tabular}{lccc}
\hline & $\begin{array}{c}\text { Isoproterenol Induced } \\
\text { AF After } \\
\text { Catheter Ablation }\end{array}$ & $\begin{array}{c}\text { Pacing Induced } \\
\text { AF After } \\
\text { Catheter Ablation }\end{array}$ & P \\
\hline Sensitivity & $33 \%$ & $44 \%$ & 0.73 \\
Specificity & $97 \%$ & $72 \%$ & 0.0002 \\
$\begin{array}{l}\text { Positive Predictive } \\
\quad \text { Value }\end{array}$ & $75 \%$ & $40 \%$ & 0.21 \\
$\begin{array}{l}\text { Negative Predictive } \\
\quad \text { Value }\end{array}$ & $84 \%$ & $76 \%$ & 0.27 \\
Diagnostic Accuracy & $83 \%$ & $64 \%$ & 0.03 \\
\hline
\end{tabular}




\section{Discussion}

\section{Main Findings}

In this study of patients with PAF, the main findings were: (1) isoproterenol induced AF in $90 \%$ of patients with PAF; (2) rechallenge with isoproterenol after termination of $\mathrm{AF}$ by ablation lead to additional ablation in $\sim 20 \%$ of patients; and (3) the post-ablation response to isoproterenol was a more accurate predictor of clinical outcome than the response to rapid atrial pacing. These findings underscore the clinical utility of noninducibility of $\mathrm{AF}$ as an endpoint of catheter ablation of PAF and demonstrate that isoproterenol infusion is preferable to rapid atrial pacing for assessing noninducibility.

\section{Isoproterenol and Inducibility of AF}

Consistent with the findings of a prior study, ${ }^{4} \mathrm{AF}$ was inducible with isoproterenol in $\sim 90 \%$ of the patients with PAF. Because AF was terminated by APVI in $65 \%$ of patients, it is likely that isoproterenol promoted PV arrhythmogenicity which initiated and perpetuated $\mathrm{AF}^{3}$ The mechanisms, by which isoproterenol induces AF may include an increase in intracellular calcium that facilitates abnormal automaticity or early after-depolarizations and triggered activity. Accentuated antagonism leading to enhanced parasympathetic tone also may play a role in the inducibility of AF by isoproterenol. $^{3}$

The persistence of AF after APVI indicates that there are residual drivers beyond the PV antrum capable of perpetuating AF. If patients undergo APVI during sinus rhythm with no attempt to assess inducibility post-ablation, the patients who require ablation beyond the PV antral regions would remain unidentified.

\section{Endpoint for Catheter Ablation of Paroxysmal AF}

Although noninducibility of AF by isoproterenol is a more accurate predictor of clinical outcomes after AF ablation than is noninducibility by rapid atrial pacing, the positive and negative predictive values of isoproterenol in this study nevertheless were $<90 \%$. Possible explanations for recurrent $\mathrm{AF}$ during follow-up despite noninducibility of $\mathrm{AF}$ by isoproterenol acutely are: (1) recovery of PV conduction during follow-up; (2) inadequate ablation of critical sites outside the PVs such that drivers or triggers reemerge after a period of stunning; and (3) the emergence of new triggers/drivers of $\mathrm{AF}$ that were not present during the index procedure.

Possible explanations for freedom from AF during followup despite the acute failure to render AF noninducible by isoproterenol or to terminate isoproterenol-induced $\mathrm{AF}$ by ablation are: (1) high-dose isoproterenol may sometimes provoke triggers or drivers that do not occur spontaneously; and (2) lesion maturation and atrial remodeling may result in eventual freedom from AF even though AF was still inducible acutely after ablation. The fact that the specificity of the response to isoproterenol was significantly better than that of rapid atrial pacing indicates that the $\mathrm{AF}$ that is induced by isoproterenol is much more likely to be a clinically relevant arrhythmia than the AF induced by rapid atrial pacing. Rapid atrial pacing is more likely to induce AF that is a nonspecific response and unlikely to occur spontaneously. Therefore, the use of rapid atrial pacing to assess the inducibility of AF may result in unnecessary additional ablation, prolongation of the procedure time, and an increase in the risk of procedural complications.

However, rapid atrial pacing does have one advantage over isoproterenol for post-ablation assessment of noninducibility. In this study, rapid atrial pacing was more sensitive than isoproterenol for identifying reentrant atrial tachycardias. Rapid atrial pacing induced an atrial tachycardia/flutter in $10 \%$ of the patients who did not have inducible AF or atrial tachycardia by isoproterenol. These patients underwent successful ablation of the tachycardia circuits and did not have any recurrences during follow-up. Therefore, although not as useful as isoproterenol for the assessment of AF noninducibility, rapid atrial pacing is clinically useful for identifying reentrant atrial tachycardias.

\section{Limitations}

In this study, catheter ablation was performed under conscious sedation. Because anesthetic agents may modify the effects of isoproterenol, the results of this study may not apply to patients who undergo general anesthesia during catheter ablation.

A variable and fairly minimal amount of CFAE ablation was performed in the patients who did not meet the procedural endpoint after APVI, and $25 \%$ of patients required transthoracic cardioversion to restore sinus rhythm. It is likely that more aggressive CFAE ablation would have resulted in more patients reaching the endpoint of AF termination and noninducibility by isoproterenol. However, some operators wanted to minimize the amount of CFAE ablation in order to preserve left atrial function as much as possible. A benefit of the variable approach to AF termination by CFAE ablation was that it allowed for analysis of the sensitivity and specificity of $\mathrm{AF}$ noninducibility for freedom from $\mathrm{AF}$ during follow-up.

This study was not a direct comparison of the predictive accuracy of inducibility of AF by rapid atrial pacing or isoproterenol in that additional ablation was performed if $\mathrm{AF}$ was reinduced with isoproterenol; however, no additional ablation was performed if $\mathrm{AF}$ was reinduced by rapid atrial pacing.

Another limitation of this study was that there were only 8 patients in the subgroup of patients in whom AF remained inducible by isoproterenol after ablation. However, this small sample size reflects the fact that the AF triggers induced by isoproterenol were successfully identified and ablated in the majority of patients.

\section{Conclusions}

Noninducibility of AF by isoproterenol infusion is a more clinically useful endpoint for catheter ablation of AF than is noninducibility of AF by rapid atrial pacing. Isoproterenol has higher specificity and better accuracy than rapid atrial pacing for predicting recurrent AF Nevertheless, rapid atrial pacing is clinically useful after catheter ablation of AF because it may uncover reentrant atrial tachycardias that then can be ablated. However, if rapid atrial pacing provokes AF after isoproterenol has failed to induce AF, the results of this study indicate that this should not prompt additional ablation. 


\section{References}

1. Haissaguerre M, Sanders P, Hocini M, Hsu LF, Shah DC, Scavee C, Takahashi Y, Rotter M, Pasquie JL, Garrigue S, Clementy J, Jais P: Changes in atrial fibrillation cycle length and inducibility during catheter ablation and their relation to outcome. Circulation 2004;109:3007-3013.

2. Oral H, Chugh A, Lemola K, Cheung P, Hall B, Good E, Han J, Tamirisa K, Bogun F, Pelosi F Jr, Morady F: Noninducibility of atrial fibrillation as an end point of left atrial circumferential ablation for paroxysmal atrial fibrillation: A randomized study. Circulation 2004;110:2797-2801.

3. Oral H, Ozaydin M, Tada H, Chugh A, Scharf C, Hassan S, Lai S, Greenstein R, Pelosi F Jr, Knight BP, Strickberger SA, Morady F: Mechanistic significance of intermittent pulmonary vein tachycardia in patients with atrial fibrillation. J Cardiovasc Electrophysiol 2002;13:645-650.
4. Oral H, Crawford T, Frederick M, Gadeela N, Wimmer A, Dey S, Sarrazin JF, Kuhne M, Chalfoun N, Wells D, Good E, Jongnarangsin K, Chugh A, Bogun F, Pelosi F Jr, Morady F: Inducibility of paroxysmal atrial fibrillation by isoproterenol and its relation to the mode of onset of atrial fibrillation. J Cardiovasc Electrophysiol 2008;19:466470.

5. Good E, Oral H, Lemola K, Han J, Tamirisa K, Igic P, Elmouchi D, Tschopp D, Reich S, Chugh A, Bogun F, Pelosi F Jr, Morady F: Movement of the esophagus during left atrial catheter ablation for atrial fibrillation. J Am Coll Cardiol 2005;46:2107-2110.

6. Nademanee K, McKenzie J, Kosar E, Schwab M, Sunsaneewitayakul B, Vasavakul T, Khunnawat C, Ngarmukos T: A new approach for catheter ablation of atrial fibrillation: Mapping of the electrophysiologic substrate. J Am Coll Cardiol 2004;43:2044-2053. 